Research Article

\title{
Characterization of Contact-Type Defects in Mortar Using a Nonlinear Ultrasonic Method
}

\author{
Zhichao Nie $\mathbb{D}^{1},{ }^{1}$ Kui Wang $\left(\mathbb{D},{ }^{1,2}\right.$ Mingjie Zhao $\mathbb{D}^{1,2}$ and Xiao Sun ${ }^{3}{ }^{3}$ \\ ${ }^{1}$ Key Laboratory of Hydraulic and Waterway Engineering of Ministry of Education, Chongqing Jiaotong University, \\ Chongqing 400074, China \\ ${ }^{2}$ Engineering Research Center of Diagnosis Technology and Instruments of Hydro-Construction, Chongqing Jiaotong University, \\ Chongqing 400074, China \\ ${ }^{3}$ School of Hydraulic and Ecology Engineering, Nanchang Institute of Technology, Nanchang 330099, China
}

Correspondence should be addressed to Kui Wang; anhuiwk@163.com

Received 7 September 2020; Revised 8 October 2020; Accepted 16 October 2020; Published 9 November 2020

Academic Editor: Zhigang Zang

Copyright (c) 2020 Zhichao Nie et al. This is an open access article distributed under the Creative Commons Attribution License, which permits unrestricted use, distribution, and reproduction in any medium, provided the original work is properly cited.

Second harmonic generation (SHG) is one of the common techniques in the nonlinear ultrasonic test. The contact-type defects play an important role in material damage, which are hard to be detected. The traditional nonlinear parameter $\beta$ used to evaluate the micro damage in material is derived from the classical stress-strain relation, which is more suitable for the anharmonicity of crystal rather than the contact-type defects. Recently, the theoretical model based on the bilinear stiffness law was derived, and the validity and applicability need to be further studied. For this purpose, by the numerical method, the contact interface in mortar is characterized based on the damage indicator $\gamma$. The relation between the excitation voltage and $\gamma$ is obtained. Moreover, the effects of the crack length and orientation on the damage indicator $\gamma$ are also obtained. The experimental method is also used to characterize the contact interface in mortar. Combining with the existing work, the results obtained in this article are discussed, and further conclusions can be drawn. The conclusions in this article provide potential of quantitative detection of the contact interface and quality evaluation of bonding layers in materials.

\section{Introduction}

The generation and development of the micro cracks lead to stress concentration, playing an important role in material deterioration and failure [1]. For decades, nonlinear ultrasonic methods have gained significant attention, and they offer enormous potential for detecting and evaluating the change in the microstructure of the materials [2-6]. A lot of studies have proved that traditional linear ultrasonic methods are infeasible for the micro damage detection in a material [6-8]. However, in this stage, the mechanism and regularity of the contact acoustic nonlinear behavior caused by interface are still insufficient, which should be studied more comprehensively.

Experimental studies on the higher harmonic generation (HHG) technique have been extensively conducted $[9,10]$, and rich results have been obtained for different damage types (crystal dislocation, precipitation, micro cracks, etc.) and materials (metals, rock, concrete, etc.) [11-14]. Initially, most studies focused on the cubic nonlinearity, which belongs to the field of classical nonlinear ultrasound. For two decades, it has been demonstrated that the nonlinearity generated by contact-type defects at the mesoscale can be orders of magnitude higher than that of the microscale $[15,16]$. This provides the possibility of damage detection for the contact interfaces. In subsequent studies, the HHG technique for contact interface detection has been extensively studied [17-19], and the nonlinear parameter $\beta$ is generally used to characterize the damage. However, there are still many barriers before applying this technique in practical test. For example, there are a lot of theoretical studies on the SHG at the contact-type defects, while the validation by the experiment is still insufficient. In addition, especially for geomaterial and concrete, the contact-type 
defects are extensively and randomly distributed, while the unified baseline of the damage state of the materials is hard to determine [20-22]. Thus, the results cannot be compared with each other to obtain further quantitative conclusions [23-25].

Theoretically, three crack-wave interaction modelling approaches (i.e., bilinear stiffness, hysteresis, and rough surface contact) are commonly used to explain the HHG phenomenon, which have been comprehensively reviewed by Broda et al. [26]. The three models have their own advantages and disadvantages. The assumption of the bilinear stiffness is hard to be satisfied, unless the level of excitation voltage is high enough to drive the cyclic open-closed behavior of the surfaces. The rough surface contact is too complex when dealing with solids with distributed microcracks. Hysteresis is a phenomenological model, and the physical structure of the crack is not taken into consideration. Recently, the scattering effect accompanied with the nonlinear ultrasonic phenomenon generating at the contact surface has attracted much attention [27-30]. Based on the rough surface contact, the nonlinear ultrasonic effects generated at the interface is studied, and the results have been verified through experiment [31]. In addition, a novel approach is proposed to analyze the scattered field at a plane interface based on the rough surface model. The corresponding solution is obtained by decomposing the scattered field into two components. The novel approach is simpler for the less unknowns [30]. Furthermore, the explicit analytical solution of the scattered field is derived, improving the previous theoretical studies obtained by numerical method [32]. In addition, Zhao et al. derived the nonclassical nonlinear parameter based on a bilinear stiffness [33, 34]. However, the proposed model is still insufficient for lack of experimental validation. Besides, $\beta_{0}$ is a global indicator, which provides no information on the local damage in materials.

In actual test, acoustic nonlinearity is commonly introduced by the electronic system as well as the coupling between the transductors and specimens. Thus, it is difficult to distinguish the material nonlinearity from the obtained nonlinearity in experiment. In general, the numerical method can be considered as an effective method for the nonlinear ultrasonic study [35-39]. By finite element simulations, the motion of interface obeys the contact laws, and the nonlinear effects of ultrasonic waves can be obtained. By FEM analysis, the nonlinear ultrasonic technique is beneficial in detecting a closed crack with a different orientation [40]. A finite difference time-domain technique is proposed to study the wave scattering caused by cracks with interacting faces, and the accuracy and stability of the scheme in one-dimensional (1D) and two-dimensional (2D) spaces are verified [41]. However, numerical simulations are difficult to consider all factors having effects on the results. Thus, considerable difference usually exists between the numerical results and the actual test, and the experiment method is necessary to be a supplement.

The objective of this study is to detect the contact interface in materials based on the bilinear stiffness assumption. For this purpose, the damage indicator derived from the bilinear stiffness laws is verified numerically. The effects of excitation voltage on the indicator $\gamma$ are investigated. Furthermore, the effects of the interface length and orientation on the second harmonic generation are also studied. The experimental study is conducted in mortar specimens, and the regularities are coincident with those of numerical results.

The paper is structured as follows. In Section 2, the theoretical background of the nonclassical damage indicator $\gamma$ is briefly introduced. Thereafter, the finite element modelling is described in Section 3, and the numerical results are given and discussed. In Section 4, the nonlinear ultrasonic test is carried out in mortar specimens and the results are presented and discussed. Finally, the conclusions of the study are presented.

\section{SHG at Contact Surface}

The explicit expression of SHG can be derived based on the bilinear stiffness assumption. Considering a longitudinal plane wave propagation in an isotropic and a linear elastic solid with Young's modulus $E_{0}$, the corresponding wave equation is given by

$$
\frac{\partial \sigma}{\partial x}=E_{0} \frac{\partial^{2} u}{\partial x^{2}}
$$

The corresponding bilinear stiffness expression can be expressed as

$$
E=E_{0}\left[1-H\left(\varepsilon-\varepsilon^{0}\right) \frac{\Delta E}{E_{0}}\right]
$$

where $\varepsilon^{0}$ is the initial static contact strain, $H(\cdot)$ is the Heaviside step function, and coefficient $\Delta E$ is the stiffness weakened by the crack interface. It is assumed that $\varepsilon>0$ for the tensile strain. $\Delta E$ is given by the following equation:

$$
\Delta E=E_{0}-\left(\frac{\mathrm{d} \sigma}{\mathrm{d} \varepsilon}\right)_{\varepsilon>0} .
$$

Substituting the bilinear stiffness expression into the $1 \mathrm{D}$ wave equation, the inhomogeneous wave equation can be obtained by

$$
\frac{E_{0}-H\left((\mathrm{~d} u / \mathrm{d} x)-\varepsilon^{0}\right)\left(E_{0}-(\mathrm{d} \sigma / \mathrm{d} \varepsilon)\right)}{\rho} \frac{\partial^{2} u}{\partial x^{2}}=\frac{\partial^{2} u}{\partial t^{2}} .
$$

Assuming that a sine wave passes through the crack at a normal direction, the corresponding boundary-initial value problem is calculated using the following equation:

$$
\left\{\begin{array}{l}
{\left[c_{L}^{2}-\frac{\Delta E}{\rho} H\left(\frac{\partial u}{\partial x}-\varepsilon^{0}\right)\right] \frac{\partial^{2} u}{\partial x^{2}}=\frac{\partial^{2} u}{\partial t^{2}}, \quad t>0, x>0,} \\
u(0, t)=A_{1} \sin \omega t \\
u(x, 0)=0 .
\end{array}\right.
$$

By solving the above equation, the amplitude of second harmonic $A_{2}$ can be expressed as 


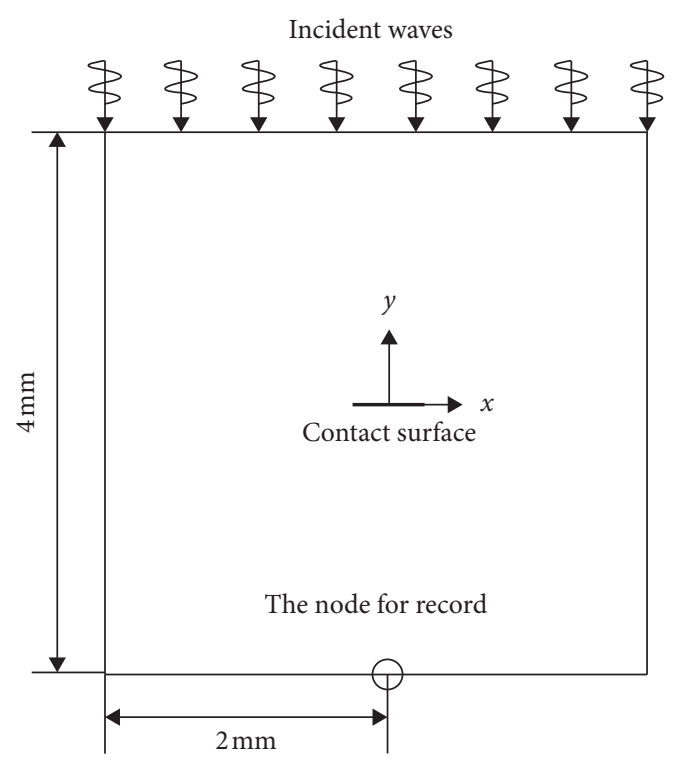

FIGURE 1: FEM model configuration.

$$
A_{2}=\frac{A_{1} \omega x}{3 \pi c_{L}},
$$

where $\omega$ is the angular frequency, $x$ is the propagation distance, and $c_{L}$ is the longitudinal velocity.

The nonclassical damage indicator $\gamma$ based on the bilinear stiffness law can be written as

$$
\gamma=\frac{A_{2}}{A_{1}}=\frac{\omega x}{3 \pi c_{L}} .
$$

It should be noted that equations (6) and (7) are established on the assumption of $\Delta E=E_{0}$ at the interface, and the tensile part of the cyclic load cannot pass through the surface in this case.

In addition, several conclusions can be drawn according to the expression of the damage indicator $\gamma$, which are as follows.

(1) The damage indicator $\gamma$ is linear with the wave propagation distance $x$ from the nonlinear source, which is the same as the classical nonlinear parameter $\beta$. The expression of $\beta$ is demonstrated in the following equation:

$$
\beta=\frac{A_{2}}{A_{1}^{2}}=\frac{k^{2} x}{8},
$$

where $k$ is the wavenumber of the incident waves.

(2) The damage indicator $\gamma$ is defined as the ratio of the second harmonic amplitude $A_{2}$ over the fundamental $A_{1}$, and the validity and reliability should be further examined.

\section{Finite Element Model}

3.1. Model Description. By defining the bilinear stiffness law at the surface, the CAN generated at the surface can be observed. In addition, the regularities of the crack length as well as the orientation on the damage indicator $\gamma$ can also be obtained. The commercial FEM software ABAQUS is used for these purposes.

Herein, a homogeneous and isotropic solid of $4 \times 4 \mathrm{~mm}^{2}$ in a $2 \mathrm{D}$ numerical simulation is considered. A contact interface of length $L_{0}$ is introduced at the center of the domain as shown in Figure 1. The cyclic load excited at the top boundary of the model is a 7-cycle Hann-windowed longitudinal pulse with the frequency of $500 \mathrm{kHz}$. The node located at the middle of the bottom boundary is selected to collect the $y$-direction displacements. The material properties are given in Table 1. The mesh of the model has a size of $0.03 \mathrm{~mm}$. This ensures that the considered wavelength $\lambda_{2 f}$ is discretized by enough elements. The element type is a 4 node bilinear plane strain quadrilateral reduced integration hourglass control (CPE4R). The mesh quality has been checked, and no poor mesh exists in the model. The time increment is $1.6 \mathrm{~ns}$, which satisfies the Courant-FriedrichsLewy stability condition, $\Delta t \leq a_{\min } \sqrt{\rho / E}\left(a_{\min }\right.$ is the smallest element dimension), and Nyquist-Shannon sampling theorem, $f_{s}>2 f_{m}\left(f_{s}\right.$ is the sampling frequency and $f_{m}$ is the maximum frequency). The total time period is $3 \times 10^{-5} \mathrm{~s}$. Fast Fourier transform (FFT) is performed to obtain the amplitudes of the fundamental $A_{1}$ and second harmonic $A_{2}$. To eliminate the nonlinearity coming from the large displacement and rotation of the elements, the geometrical nonlinearity is set to be off, and both the linear and the quadratic bulk viscosity parameters are zero. Therefore, it can be concluded that the detected nonlinearity is solely generated by the contact behavior of the interface surfaces.

3.2. Contact Laws. The bilinear stress-strain law is assumed to be concerned with the stiffness asymmetry at the crack surfaces, which can be stated as

$$
\sigma= \begin{cases}\left(E_{0}-\Delta E\right) \mathcal{E}, & \varepsilon>\varepsilon_{0}, \\ E_{0} \varepsilon, & \varepsilon \leq \varepsilon_{0} .\end{cases}
$$

It is assumed that $\varepsilon>0$ is positive for the tensile strain. The weakened elastic modulus $\Delta E$ is dependent on the damage degree in material. When the interface is well bonded (i.e., no damage exists in material), $\Delta E$ equals 0 in the cyclic compression-tensile load. When the interface is ruptured, $\Delta E$ will increase to $E_{0}$, and the tensile components of the cyclic load are unable to pass through the surface. In this numerical model, the contact laws applied at the crack can be stated as

$$
\sigma= \begin{cases}0, & \varepsilon>0, \\ E_{0} \varepsilon, & \varepsilon \leq \varepsilon_{0} .\end{cases}
$$

The above contact laws are coincident with the theoretical background. The prestress $\sigma_{0}$ is assumed to be zero in the model. In addition, it should be noted that the second harmonic generation depends on the asymmetrical normal stiffness. For consistency, the surface is considered flat and frictionless, and the vertical displacement is received and analyzed. In addition, the contact type of the interface is 
TABLe 1: Material properties of the FEM model.

\begin{tabular}{lcccc}
\hline Material & Density $\rho\left(\mathrm{kg} / \mathrm{m}^{3}\right)$ & Young's modulus $E(\mathrm{GPa})$ & Poisson's ratio $v$ & Friction coefficient $\mu$ \\
\hline Mortar & 2200 & 21.1 & 0.167 & 0 \\
\hline
\end{tabular}

"hard contact." This indicates that the surfaces cannot interpenetrate into each other, and the crack surface allows separation after the contact.

The time and frequency domain spectra of the intact and cracked models are shown in Figure 2. The amplitude of the excitation is $80 \mathrm{~nm}$ in this case, and the length of the crack is $1 \mathrm{~mm}$. The elastic waves are normally incident at the interface. It can be concluded that, for the cracked sample, the $+y$ direction displacement of the bottom element of the surface is obviously small. This indicates that the tensile strain is hard to pass through the unbonded interface. It can be observed from the frequency spectrum that significant second harmonic appears at the surface of the crack compared with the intact sample. As mentioned above, the finite element model has no geometric and material nonlinearity, and the acoustic nonlinearity should come from the interaction between the surfaces of the crack. The above results further prove that the contact laws are effective, which can be further applied in the numerical study.

\subsection{Numerical Results}

3.3.1. Effects of the Excitation Levels on $\gamma$. As introduced in the theoretical background, the damage indicator $\gamma$ is defined as the ratio of the second harmonic amplitude $A_{2}$ to the fundamental $A_{1}$, and the damage indicator $\gamma$ is a constant with increase of the excitation levels. The FEM analysis will be conducted to verify the analytical expression.

The amplitude of the incident waves varies from 10 to $150 \mathrm{~nm}$ with a step of $10 \mathrm{~nm}$. In addition, the crack has a length of $1 \mathrm{~mm}$ and is normal to the incident waves. The damage indicator $\gamma$ for various excitation levels is presented in Figure 3. It can be noted that when the amplitude of incident wave is weak, corresponding $\gamma$ is small. With the amplitude increasing, $\gamma$ increases sharply. Then, $\gamma$ remains stable in case of large enough amplitude applied. It is known that the CAN effects will occur once the excitation level becomes greater than the threshold. The strong cyclic load near the surface will lead the nonlinear effects. Considering that the trend of $\gamma$ is in accordance with the CAN model, the correctness and validity of $\gamma$ can be examined by the FEM model.

\subsubsection{Effects of the Interface Length on the Damage Indicator} $\gamma$. The length of contact interface is characterized based on the damage indicator $\gamma$. The crack length $L_{0}$ is arranged from 0.4 to $1 \mathrm{~mm}$ with an interval of $0.1 \mathrm{~mm}$. The incident waves are normally excited at the top surface of the model. The damage indicator $\gamma$ is determined by the linear fitting of $A_{1}$ and $A_{2}$. The amplitude of the incident waves varies from 60 to $100 \mathrm{~nm}$ with a step of $10 \mathrm{~nm}$. The damage indicator $\gamma$ for various crack lengths is shown in Figure 4 . As can be seen in Table 2, the coefficient of determination $R^{2}$ of each fit line is nearly 1 , which indicates that the excitation level is independent of the damage indicator $\gamma$. In addition, Figure 5 shows that the crack length has a significant effect on the damage indicator $\gamma$. A larger damage indicator $\gamma$ will be obtained with increase of the interface length. A significant positive correlation between the damage indicator $\gamma$ and the crack length $L_{0}$ can be obtained.

It is worth mentioning that the crack length is also characterized by other damage indicators based on the nonlinear ultrasonic method [34], as shown in Figure 6. It can be concluded that the nonlinear effects are stronger when the crack becomes longer. Thus, the FEM results provide potential of quantitively characterizing the contact interface in materials.

\subsubsection{Effects of the Interface Angle on the Damage Indicator} $\gamma$. To understand the regularity of the crack orientation on the damage indicator $\gamma$, seven crack orientations $\left(\alpha=0^{\circ}, 15^{\circ}\right.$, $30^{\circ}, 45^{\circ}, 60^{\circ}, 75^{\circ}$, and $90^{\circ}$ ) are considered. The crack length is $1 \mathrm{~mm}$ in this section. Besides, the amplitude of the incident waves varies from 60 to $100 \mathrm{~nm}$ with a step of $10 \mathrm{~nm}$. Similarly, the damage indicator $\gamma$ is determined by linear fitting of $A_{1}$ and $A_{2}$. With increase of the excitation level, the distribution of indicator $\gamma$ can be observed in Figure 7 . Figure 8 shows the damage indicator $\gamma$ versus the crack orientation. It can be concluded that the damage indicator $\gamma$ decreases as the crack angle increases. According to the bilinear stiffness assumption, the second harmonic is generated by the clapping effect of the surface. Thus, the significant contact nonlinearity should appear in case of the waves being normally incident at the surface.

In other studies, the similar regularity of the crack orientation on the second harmonic generation has been obtained [35]. As shown in Figure 9, in the case of $\mathrm{P}$ wave, normalized $A_{1}$ and $A_{2}$ are affected by the crack angle. Fundamental $A_{1}$ increases with the interface angle, while the opposite trend can be observed on the second harmonic $A_{2}$. Thus, the regularity of the damage indicator $\gamma$ decreases with increase of the interface angle, which is consistent with the numerical results in this paper.

\section{Experimental Procedure}

4.1. Sample Preparation. The size of the mortar specimens is $100 \mathrm{~mm} \times 100 \mathrm{~mm} \times 100 \mathrm{~mm}$. Three groups of cubic mortar specimens were prepared in this experiment. Group A contains 3 intact mortar specimens (I1 I3), which is used to study the relation between the excitation voltage and the damage indicator $\gamma$. Group B contains 6 specimens (L1 L6). The length of the interface is $1 \sim 6 \mathrm{~cm}$, with an interval of $1 \mathrm{~cm}$. The interface angle is $0^{\circ}$ in this group. Group $\mathrm{C}$ contains 7 specimens (A1 A7). The crack orientation is $0 \sim 90^{\circ}$ with a step of $15^{\circ}$. The interface length is $4 \mathrm{~cm}$. The 


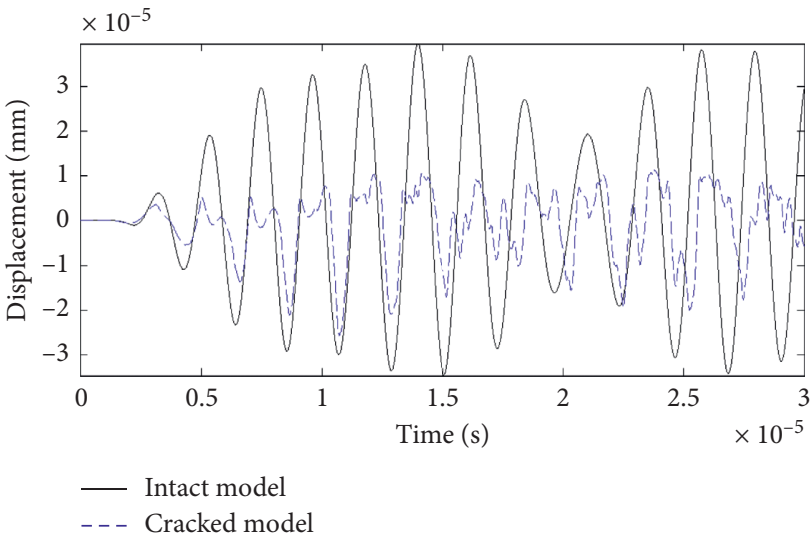

(a)

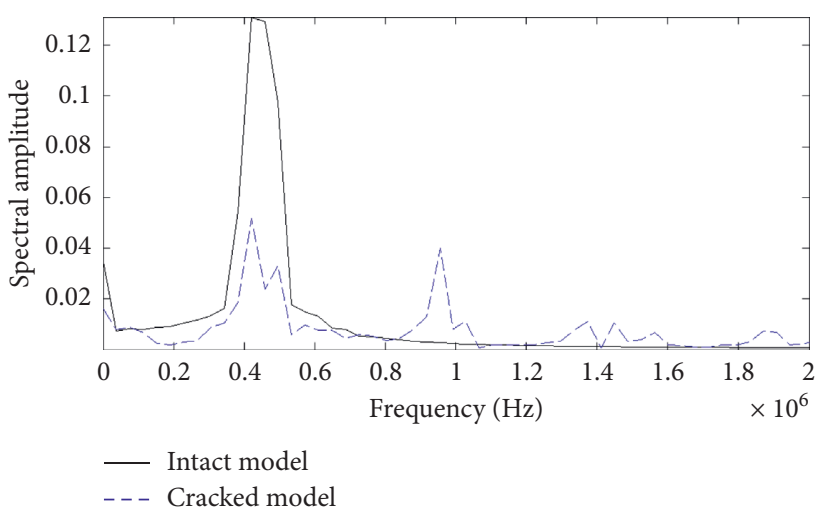

(b)

Figure 2: (a) Time domain. (b) Frequency domain spectra comparison of the intact and the cracked numerical models.

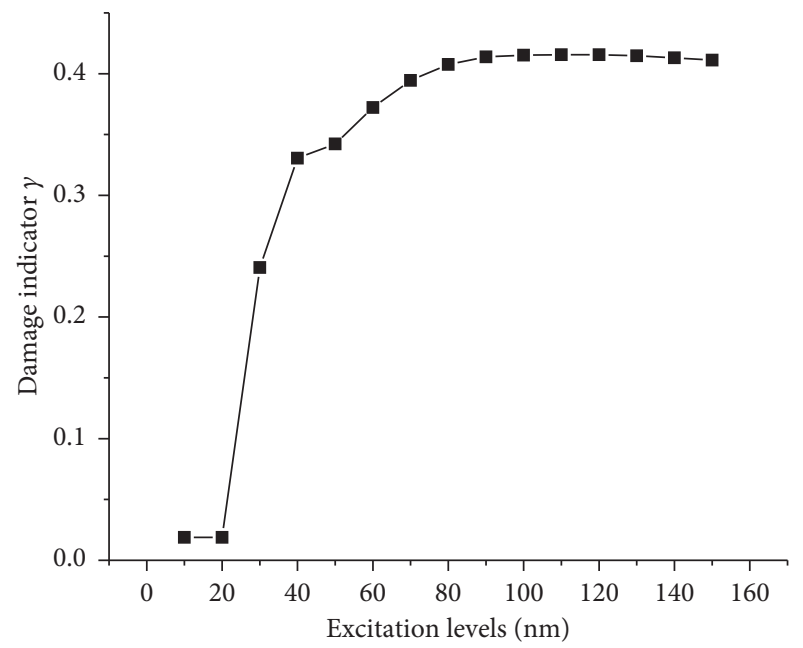

FIgURE 3: Damage indicator $\gamma$ for various excitation levels.

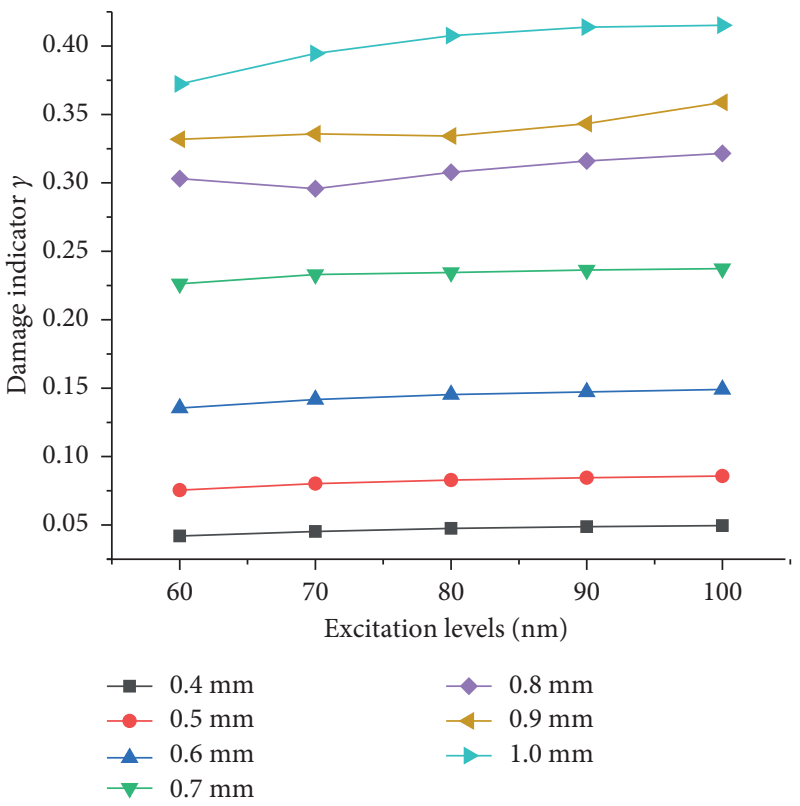

FIgURE 4: Damage indicator $\gamma$ for various crack lengths.
TABLE 2: The coefficient of determination $R^{2}$ for the linear fitting of $A_{1}$ and $A_{2}$.

\begin{tabular}{lccccccc}
\hline Crack length $(\mathrm{mm})$ & 0.4 & 0.5 & 0.6 & 0.7 & 0.8 & 0.9 & 1 \\
\hline $\begin{array}{l}\text { Coefficient of } \\
\text { determination } R^{2}\end{array}$ & 0.999 & 1 & 1 & 1 & 0.995 & 0.993 & 0.998
\end{tabular}

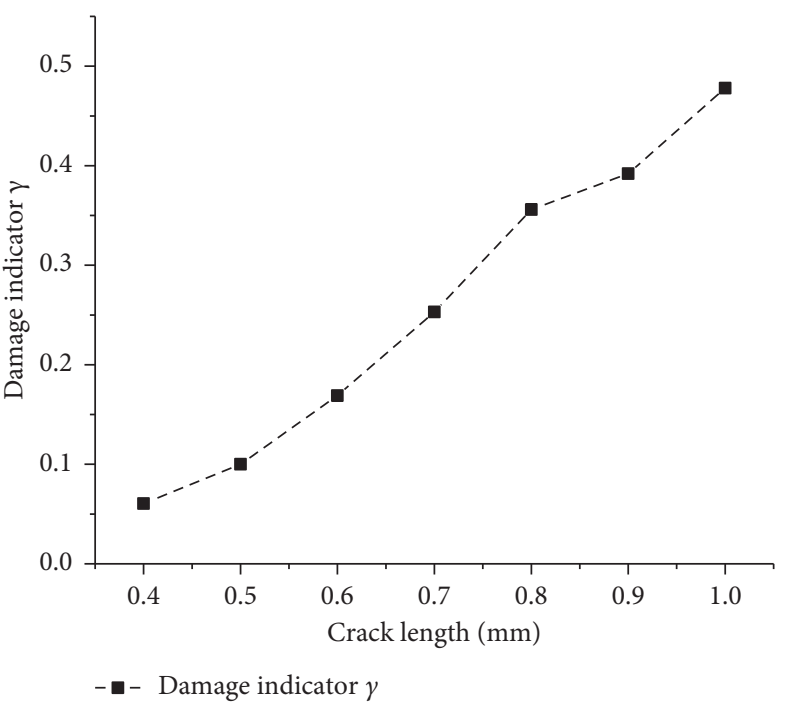

FIGURE 5: Damage indicator $\gamma$ for various crack lengths.

introduction procedure of the contact interface will be given later. The mixture proportions and physical properties of the mortar specimens are given in Table 3 . The Portland cement (P.O. 32.5) was used in this experiment.

The procedure for the contact interface generation is as follows. After pouring of mortar, a thin steel sheet with machine oil on the surface vertically penetrated into the mortar mixture. All the steel sheets are $12 \mathrm{~cm}$ in length and $1 \mathrm{~mm}$ in thickness. The insertion depth is $100 \mathrm{~mm}$. The width of the steel sheets is $1 \sim 6 \mathrm{~cm}$. Then, the steel sheet was removed out of the specimen carefully, and the specimens were not moved before being demolded. It should be noted that the removal of the steel sheets was gradually conducted 


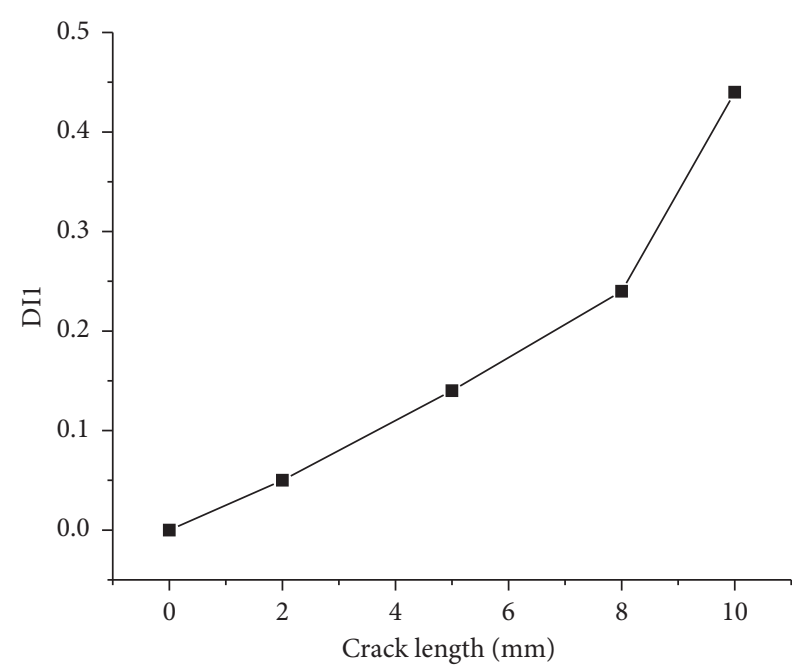

Figure 6: Damage index DI1 versus the crack lengths [34].

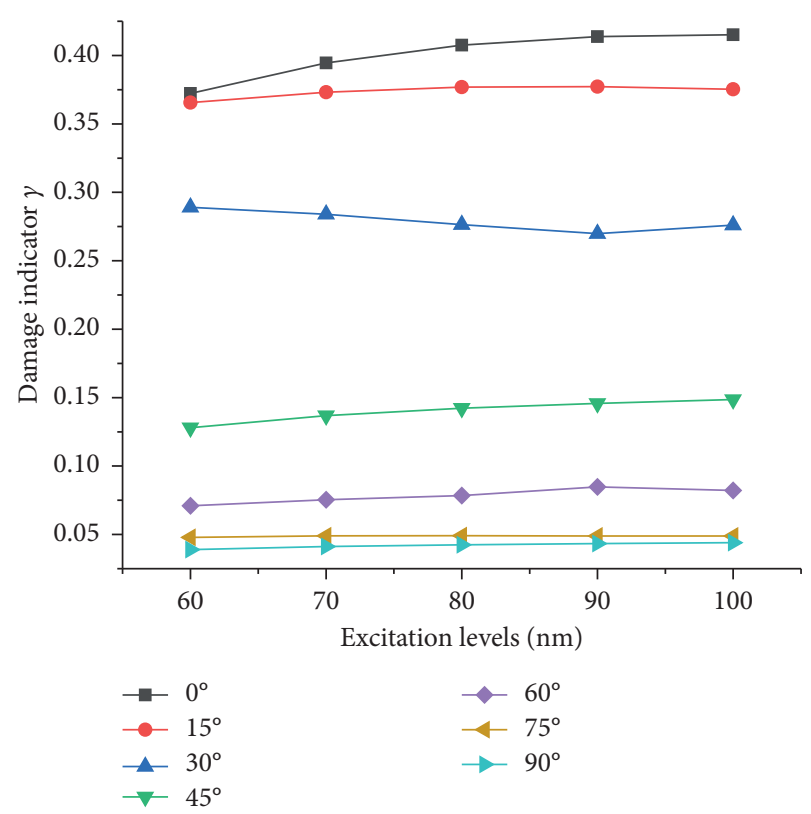

Figure 7: Amplitude of fundamental $A_{1}$ versus second harmonic $A_{2}$ for various crack angles.

once sheets arrived at the bottom of the sample, and the whole process of interface introduction took about one minute. Consequently, an artificial contact interface was left in the specimen due to the fluidity of the mixture. The specimens were demolded after $24 \mathrm{~h}$ followed by curing in a standard chamber $\left(95 \%\right.$ relative humidity and $\left.20^{\circ} \mathrm{C}\right)$ until 28 days of age. The mortar specimens are shown in Figure 10.

To confirm that the artificial interface is contact, the pulse velocity of each specimen is tested, and the related results are shown in Figure 11. It is concluded that the wave velocity remains steady in all specimens, which indicates that the contact interface is at micro scale and cannot be detected by the traditional ultrasonic method. Moreover, the specimen A7 was cut in two halves after the test, as shown in Figure 12. The trace along the height of specimen can be

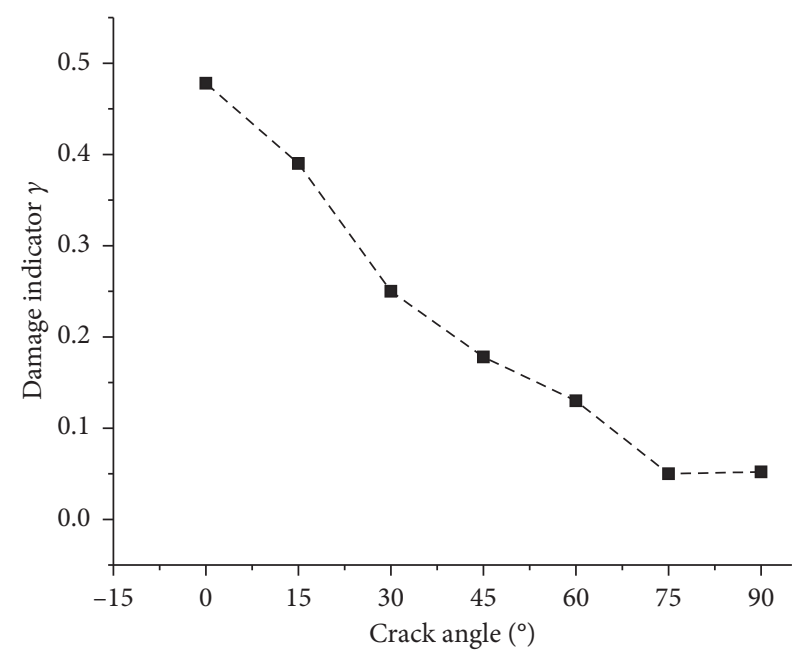

FIgURE 8: Distribution of damage indicator $\gamma$ for various crack angles.

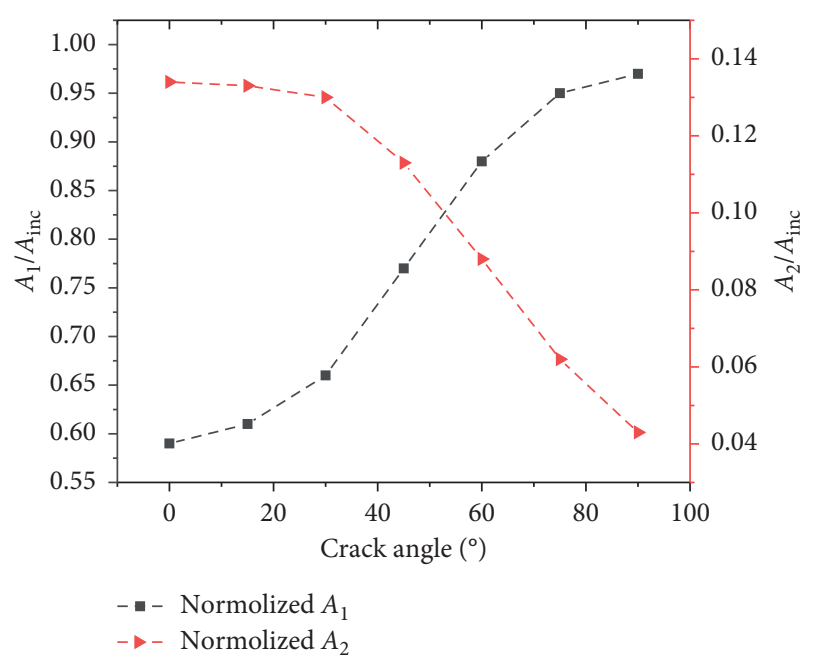

Figure 9: Distribution of fundamental $A_{1}$ and second harmonic $A_{2}$ for various crack angles [35].

observed. It is noted that the damage degree gradually weakened along the height of specimen because of the selfweight of the mixture. In conclusion, the interface within the specimen can be considered contact, and the damage is essentially at micro scale.

It should be pointed out that equation (8) is established on the assumption of the surface rupture, while the interface within the mortar can be considered the stiffness reduction, and the corresponding analytical expression can be given as [42]

$$
A_{2}=\frac{\left(\gamma_{t}-\gamma_{c}\right) A_{1} x \omega}{3 \pi c_{L}}
$$

where $\gamma_{t}$ and $\gamma_{c}$ represent the tension and compression asymmetry in the elastic modulus, respectively. The same damage indicator can be obtained in equations (8) and (11), which is defined as the ratio of $A_{2}$ to $A_{1}$. 
TABLE 3: Details of mixture proportions and physical properties of the mortar specimens.

\begin{tabular}{lccccccc}
\hline \multirow{2}{*}{ Design compressive strength $(\mathrm{MPa})$} & \multirow{2}{*}{ Average density $\left(\mathrm{kg} / \mathrm{m}^{3}\right)$} & \multirow{2}{*}{$\mathrm{w} / \mathrm{c}(\%)$} & \multicolumn{4}{c}{ Unit quantity $\left(\mathrm{kg} / \mathrm{m}^{3}\right)$} & \multirow{2}{*}{ Average P wave velocity $(\mathrm{m} / \mathrm{s})$} \\
\hline 10 & 1984 & 60 & 336 & 560 & 1680 & 3305 \\
\hline
\end{tabular}

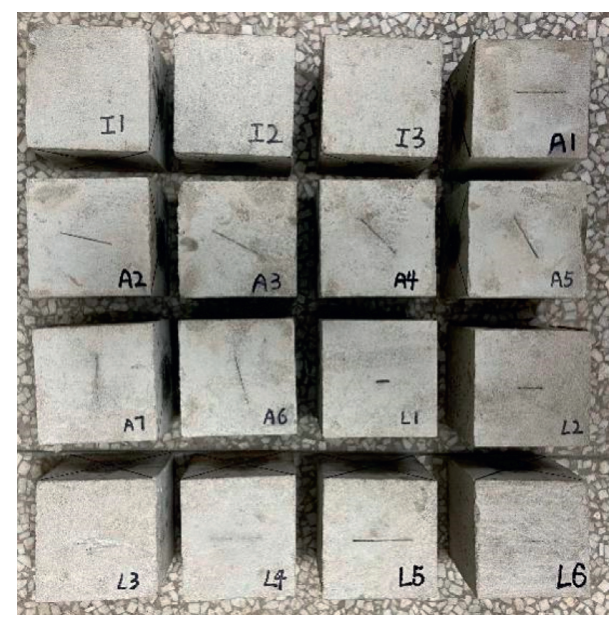

Figure 10: Mortar specimens for SHG test in experiment.

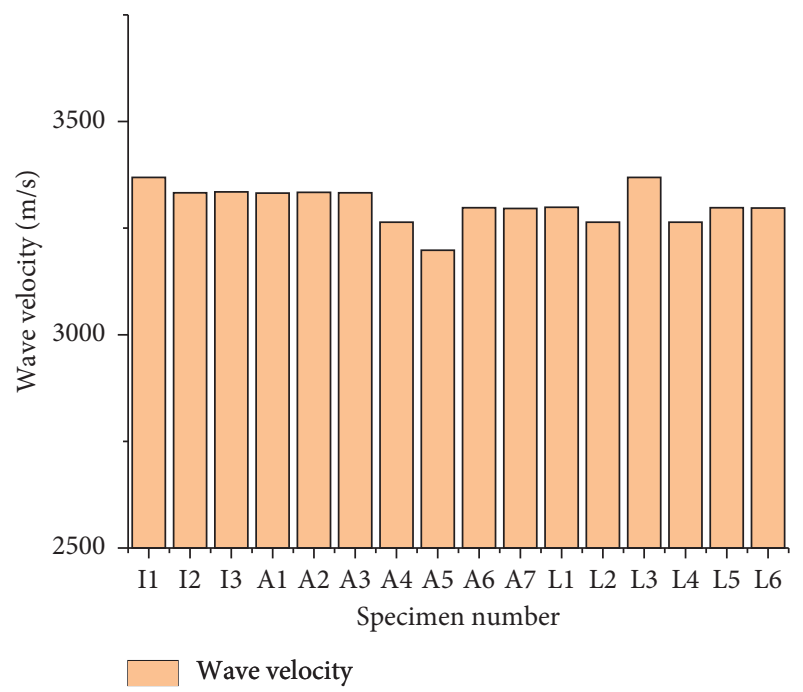

FIGURE 11: Distribution of wave velocity in each specimen.

4.2. Experimental Setup. The experimental setup for the measurement of fundamental $A_{1}$ and second harmonic $A_{2}$ is shown in Figure 13. According to the previous study [43], for mortar specimens, strong attenuation will happen when the center frequency is above $500 \mathrm{kHz}$. Therefore, a tone-burst signal of 10 cycles at $200 \mathrm{kHz}$ is generated by a function generator (Rigol 1022U). The pulse repetition rate is $10 \mathrm{~ms}$. Two contact-type transducers (PXR 50) are used at the transmitting and receiving ends. Figure 14 shows that PXR 50 has a good response to fundamental $A_{1}$ and second harmonic $A_{2}$ considering that the sensitivity at $f_{0}$ and $2 f_{0}$ is above $60 \mathrm{~dB}$. The receiving time domain signal after modulating is recorded by a digital oscilloscope (Tektronix MDO 3104), and the sampling rate is $50 \mathrm{~ms} / \mathrm{s}$. The quality and repeatability of

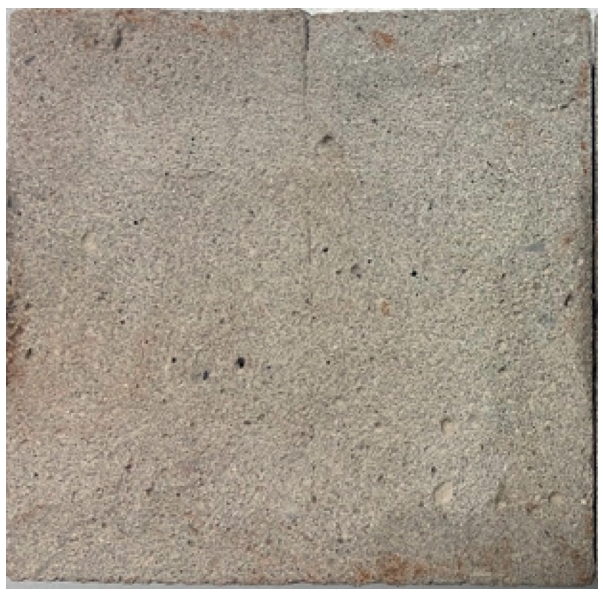

FIGURE 12: Longitudinal section diagram of the specimen A7.

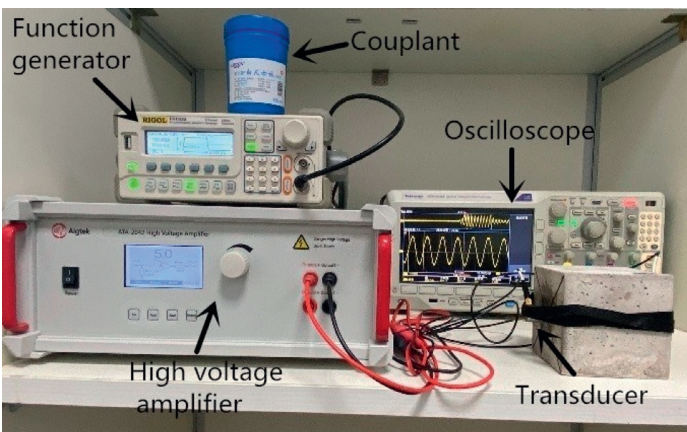

FIgURE 13: Test system for signal generation and acquisition.

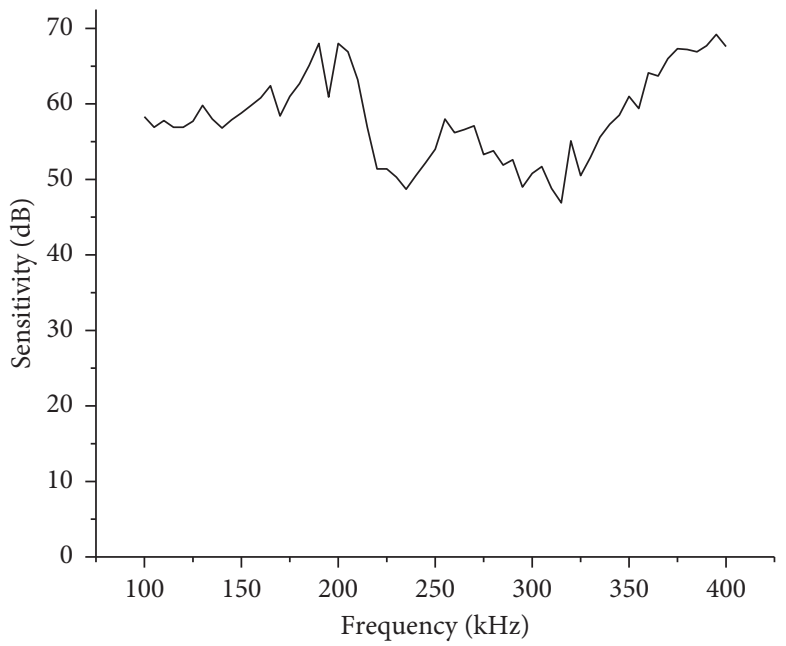

FIGURE 14: Schematic diagram of frequency response of PXR 50 transducer. 


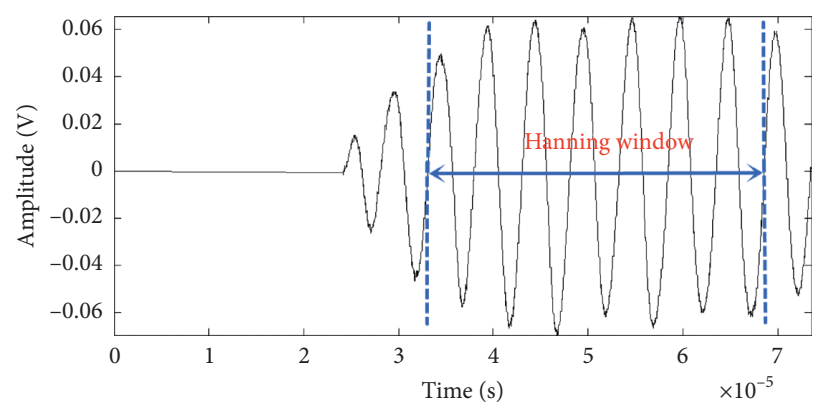

(a)

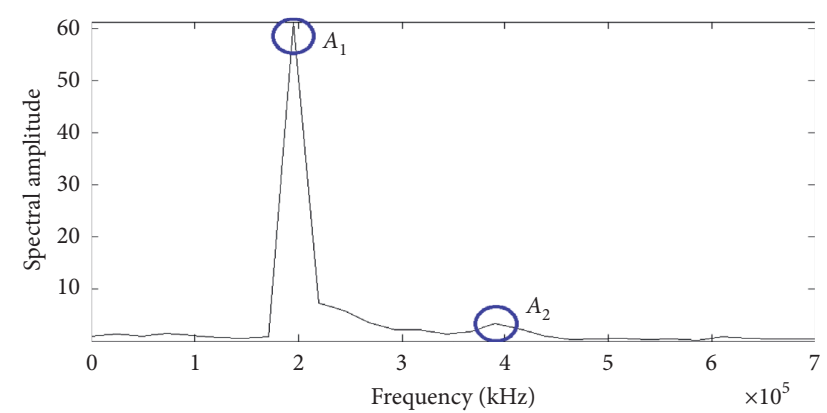

(b)

FIgURE 15: Example of the SHG test. (a) Time-domain signal. (b) Frequency-domain signal.

the measurements are improved by averaging the signals with 256 acquisitions. In addition, a thin layer of Vaseline was used as couplant between the transducers and the specimen surface. The surface of the specimens was wiped carefully to ensure no sand or dust is attached on the test point. The specimen and transducers were tightly wrapped during the test. To ensure that the transmitter and the receiver are aligned, the test points were marked before the fixation of transducers. The FFT transform was performed on the time spectrum to obtain fundamental $A_{1}$ and second harmonic $A_{2}$. Figure 15 shows an example of the time and frequency spectra measured from a mortar specimen with interface, respectively. Fundamental $A_{1}$ and second harmonic $A_{2}$ can be observed from the frequency spectrum, and the damage indicator $\gamma$ can be calculated.

\subsection{Experimental Results and Discussion}

4.3.1. Effects of the Excitation Levels on $\gamma$. The results of the intact mortar specimens are shown in Figure 16. The value of $\gamma$ is independent of the excitation voltage. In addition, the damage indicator $\gamma$ in specimens I $\sim$ I 3 can be determined by the slope of the fit line, being 0.050, 0.043, and 0.052, respectively. The damage indicator $\gamma$ in specimens I1 I3 can be regarded as the intrinsic nonlinearity of the mortar, which can be used to distinguish the nonlinearity coming from the contact interface in group B and group C.

The value of $\gamma$ is independent of the excitation voltage, which has been observed by analyzing the leaky Rayleigh wave passing through the crack surface [44]. As shown in Figure 17, the ratio of second harmonic $A_{2}$ to fundamental $A_{1}$ is almost a constant when $\sigma$ is $37.1 \mathrm{kPa}$, which indicates that the damage indicator $\gamma$ is independent of the excitation voltage. It should be noted that when a large $\sigma$ is applied, a minor gap exists between the two blocks. In view of the damage type being the same in the two studies, the results are consistent with each other even if the materials used in the studies are different. Besides, the mechanism of second harmonic generation is not explained theoretically in Vergara et al.'s study.

\subsubsection{Effects of the Interface Length on the Damage Indicator} $\gamma$. The distribution of damage indicator $\gamma$ in different interface lengths is shown in Figure 18. $\gamma_{0}$ and $\delta$ represent the

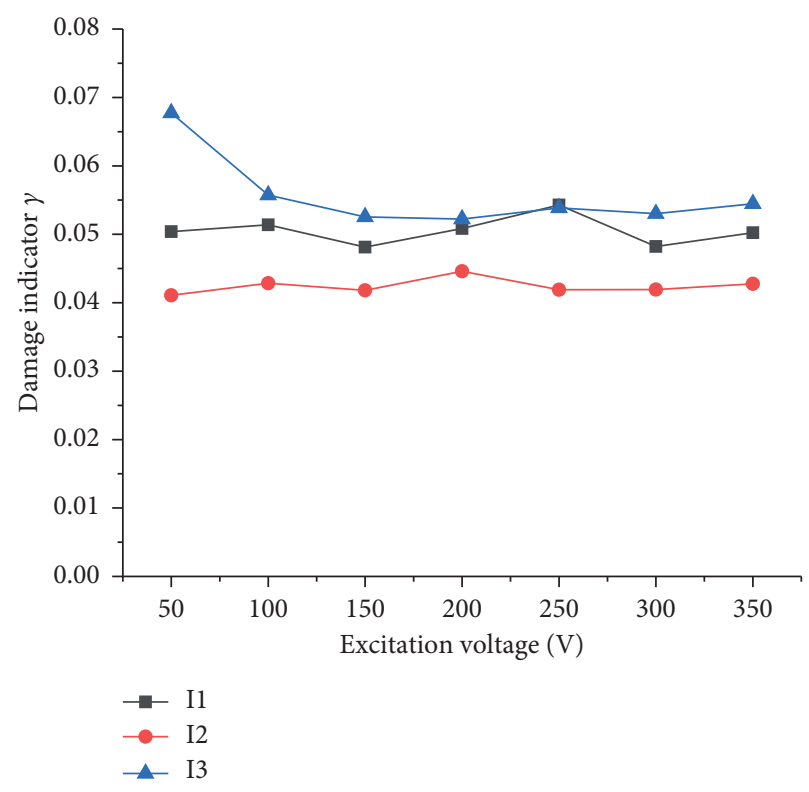

FIGURE 16: Distribution of the excitation voltage and the damage indicator $\gamma$ in specimens I1 I3.

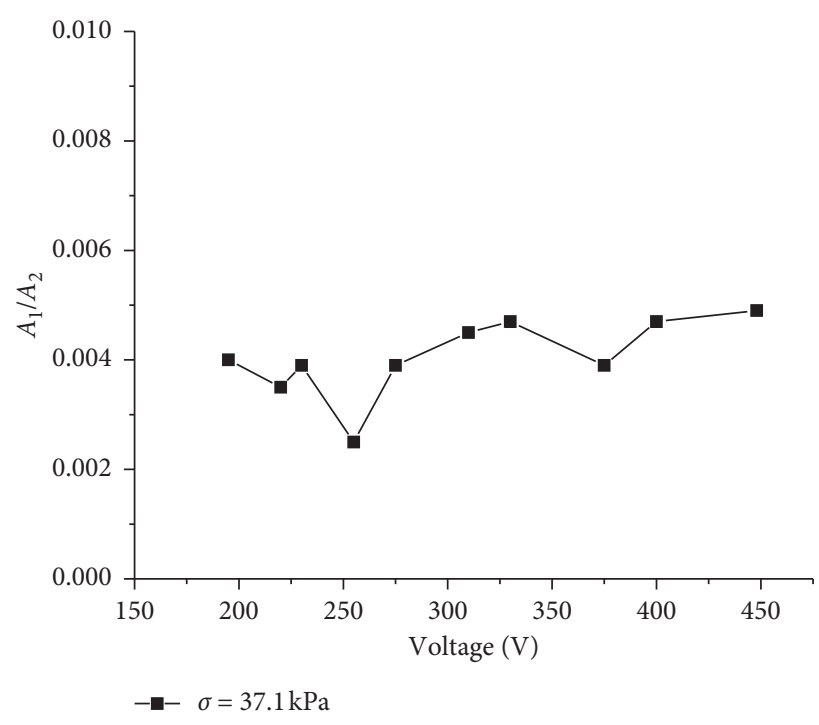

FIGURE 17: Damage indicator $\gamma$ versus the excitation voltage [44]. 


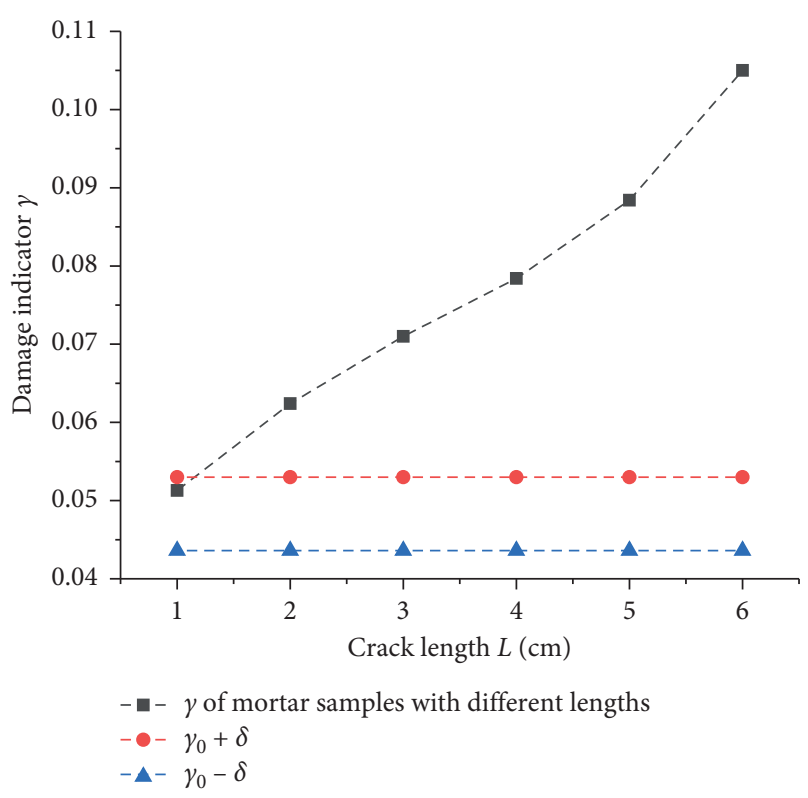

Figure 18: Damage indicator $\gamma$ in specimens L1 L3.

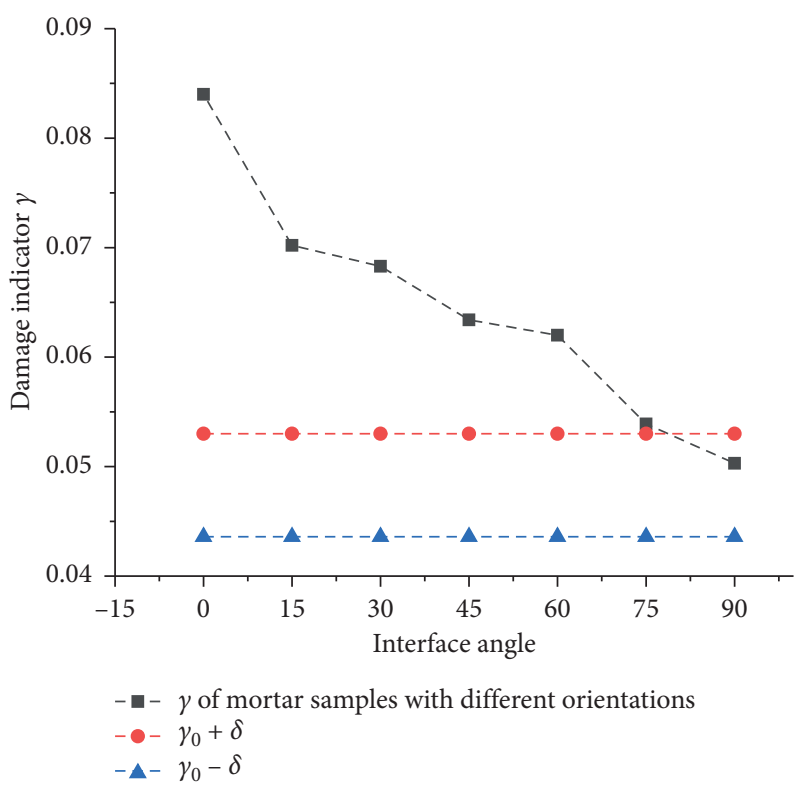

Figure 19: Damage indicator $\gamma$ in specimens A1 A7.

means and standard deviations of the damage indicator $\gamma$ in intact mortar specimens. $\gamma_{0} \pm \delta$ is used to distinguish which damage indicators of the mortar specimens with interface behave different from the intact specimens. It can be obtained that the damage indicator $\gamma$ is obviously larger than those of intact specimens for the $2-6 \mathrm{~cm}$ interface length. For the $1 \mathrm{~cm}$ interface, although the damage indicator $\gamma$ is in the band, it is still larger than the means of $\gamma$ in intact mortar specimens. Thus, it can be concluded that the damage indicator $\gamma$ is effective to characterize the contact interface in materials. On the other hand, positive correlation between the nonlinear parameter $\gamma$ and the interface length $L$ can also be revealed, which is coincident with the numerical results.
4.3.3. Effects of the Interface Angle on the Damage Indicator $\gamma$. Figure 19 shows the distribution of the damage indicator $\gamma$ in the mortar specimens with different interface orientations. The meanings of $\gamma_{0}$ and $\delta$ are the same as in the previous section. In general, the damage indicator $\gamma$ is significantly affected by the interface angle. The decreasing trend can be observed obviously with the increase of the interface angle, which is consistent with the numerical results. When the interface degree is close to $90^{\circ}$, the difference between the intact and the interface becomes smaller. In actual applications, it is reasonable to conduct the test at different direction of the material to avoid missing the interface paralleling the propagation direction of ultrasound.

\section{Conclusions}

In this paper, the SHG technique is studied based on the bilinear stiffness assumption. The main conclusions drawn in this paper can be stated as follows:

(1) By the numerical and experimental method, the regularity of the excitation levels on the damage indicator $\gamma$ is attained. Both numerical and experimental results show that $\gamma$ is a constant when the amplitude is large. In addition, $\gamma$ is effective in characterizing the contact-type defects in mortar.

(2) The regularity of the interface length on the indicator $\gamma$ is also studied. The damage indicator $\gamma$ becomes larger when the elastic waves are normally incident at the surface. It should be noted that the value of $\gamma$ is also affected by the attenuation at the surface, and the absorption and scattering effects should be considered to obtain the quantitative results.

(3) The damage indicator $\gamma$ is effective in characterizing the interface orientation. $\gamma$ decreases with the increase of the interface angle. Moreover, the results are discussed with the existing work and can be further verified and analyzed.

Further in-depth research should be carried out on the experimental studies of the CAN generation in materials, especially the validation for the existing theoretical results. In addition, quantitative characterization should be further studied, which will be of benefit to applying this technique in practical test.

\section{Data Availability}

The data used to support the findings of this study are available from the corresponding author upon request.

\section{Conflicts of Interest}

The authors declare that they have no conflicts of interest.

\section{Acknowledgments}

This research was funded by Chongqing Research Program of Basic Research and Frontier Technology, Grant no. cstc2017jcyjBX0066; Key Laboratory of Hydraulic and 
Waterway Engineering of the Ministry of Education, Grant no. SLK2017B05; Innovative Research Program of Chongqing Jiaotong University, Grant no. 2019B0101; Jiangxi Youth Science Fund Project, Grant no. 20171BAB216042; Advantage Technology Innovation Team of Jiangxi Province (Grant no. 20171BCB24012); and Science and Technology Projects of Department of Transportation of Jiangxi Province, Grant no. 2018 Q0028.

\section{References}

[1] A. Klepka, W. Staszewski, R. Jenal, M. Szwedo, J. Iwaniec, and T. Uhl, "Nonlinear acoustics for fatigue crack detection-experimental investigations of vibro-acoustic wave modulations," Structural Health Monitoring: An International Journal, vol. 11, no. 2, pp. 197-211, 2012.

[2] Y. Yang, C.-T. Ng, and A. Kotousov, "Influence of crack opening and incident wave angle on second harmonic generation of Lamb waves," Smart Materials and Structures, vol. 27, no. 5, Article ID 055013, 15 pages, 2018.

[3] H. Yan, C. Xu, D. Xiao, and H. Cai, "Properties of GH4169 superalloy characterized by nonlinear ultrasonic waves," Advances in Materials Science and Engineering, vol. 2015, Article ID 457384, 9 pages, 2015.

[4] Z. Su, C. Zhou, M. Hong, L. Cheng, Q. Wang, and X. Qing, "Acousto-ultrasonics-based fatigue damage characterization: linear versus nonlinear signal features," Mechanical System and Signal Processing, vol. 45, no. 1, pp. 1-10, 2014.

[5] C. Yang and J. Chen, "Fully noncontact nonlinear ultrasonic characterization of thermal damage in concrete and correlation with microscopic evidence of material cracking," Cement Concrete Research, vol. 123, Article ID 105797, 10 pages, 2019.

[6] J. Chen, C. Yang, and Q. Guo, "Evaluation of surface cracks of bending concrete using a fully non-contact air-coupled nonlinear ultrasonic technique," Materials and Structures, vol. 51, Article ID 104, 9 pages, 2018.

[7] M. Zhao, Z. Nie, K. Wang, P. Liu, and X. Zhang, "Nonlinear ultrasonic test of concrete cubes with induced crack," Ultrasonics, vol. 97, pp. 1-10, 2019.

[8] C. Mondal, A. Mukhopadhyay, and R. Sarkar, "A study on precipitation characteristics induced strength variation by nonlinear ultrasonic parameter," Journal of Applied Physics, vol. 108, Article ID 124910, 7 pages, 2010.

[9] M. F. Müller, J.-Y. Kim, J. Qu, and L. J. Jacobs, "Characteristics of second harmonic generation of Lamb waves in nonlinear elastic plates," The Journal of the Acoustical Society of America, vol. 127, no. 4, pp. 2141-2152, 2010.

[10] J. Kim, D.-G. Song, and K.-Y. Jhang, "Absolute measurement and relative measurement of ultrasonic nonlinear parameters," Research in Nondestructive Evaluation, vol. 28, no. 4, pp. 211-225, 2017.

[11] M. A. Breazeale and D. O. Thompson, "Finite-amplitude ultrasonic waves in aluminum," Applied Physics Letters, vol. 3, no. 5, pp. 77-78, 1963.

[12] A. Hikata, B. B. Chick, and C. Elbaum, "Effect of dislocations on finite amplitude ultrasonic waves in aluminum," Applied Physics Letters, vol. 3, no. 11, pp. 195-197, 1963.

[13] M. Fukuda and K. Imano, "Second harmonic ultrasonic waves detection using a double-layered piezoelectric transducer," Journal of the Acoustical Society of America, vol. 140, no. 4, Article ID 3326, 13 pages, 2016.
[14] P. B. Nagy, "Fatigue damage assessment by nonlinear ultrasonic materials characterization," Ultrasonics, vol. 36, no. 1-5, pp. 375-381, 2018.

[15] L. A. Ostrovsky, S. N. Gurbatov, and J. N. Didenkulov, "Nonlinear acoustics in nizhni novgorod (A review)," Acoustical Physics, vol. 51, no. 2, pp. 114-127, 2005.

[16] I. Y. Solodov, N. Krohn, and G. Busse, "CAN: an example of non-classical acoustic nonlinearity in solids," Ultrasonics, vol. 40, no. 1-8, pp. 621-625, 2002.

[17] N. Kim, T.-H. Lee, K.-Y. Jhang, and I.-K. Park, "Nonlinear behavior of ultrasonic wave at crack," AIP Conference Processing, vol. 1211, no. 1, pp. 313-318, 2010.

[18] Y. Yang, C.-T. Ng, and A. Kotousov, "Bolted joint integrity monitoring with second harmonic generated by guided waves," Structural Health Monitoring, vol. 18, no. 1, pp. 193-204, 2019.

[19] V. E. Nazarov and A. M. Sutin, "Nonlinear elastic constants of solids with cracks," The Journal of the Acoustical Society of America, vol. 102, no. 6, pp. 3349-3354, 1997.

[20] J. Chen, Y. Wu, and C. Yang, "Damage assessment of concrete using a non-contact nonlinear wave modulation technique," NDT \& E International, vol. 106, pp. 1-9, 2019.

[21] P. Antonaci, C. L. E. Bruno, A. S. Gliozzi, and M. Scalerandi, "Monitoring evolution of compressive damage in concrete with linear and nonlinear ultrasonic methods," Cement and Concrete Research, vol. 40, no. 7, pp. 1106-1113, 2010.

[22] J. C. Ongpeng, A. W. C. Oreta, S. Hirose, and K. Nakahata, "Nonlinear ultrasonic investigation of concrete with varying aggregate size under uniaxial compression loading and unloading," Journal of Materials in Civil Engineering, vol. 29, no. 2, Article ID 04016210, 7 pages, 2017.

[23] P. Liu, H. Sohn, and B. Park, "Baseline-free damage visualization using noncontact laser nonlinear ultrasonics and state space geometrical changes," Smart Materials and Structures, vol. 25, Article ID 065036, 12 pages, 2015.

[24] A. A. Shah and Y. Ribakov, "Non-linear ultrasonic evaluation of damaged concrete based on higher order harmonic generation," Materials \& Design, vol. 30, no. 10, pp. 4095-4102, 2009.

[25] T. Ju, J. D. Achenbach, L. J. Jacobs, M. Guimaraes, and J. Qu, "Ultrasonic nondestructive evaluation of alkali-silica reaction damage in concrete prism samples," Materials and Structures, vol. 50, Article ID 60, 13 pages, 2017.

[26] D. Broda, W. J. Staszewski, A. Martowicz, T. Uhl, and V. V. Silberschmidt, "Modelling of nonlinear crack-wave interactions for damage detection based on ultrasound-a review," Journal of Sound and Vibration, vol. 333, no. 4, pp. 1097-1118, 2014.

[27] L. R. F. Rose, P. Blanloeuil, M. Veidt, and C. H. Wang, "Analytical and numerical modelling of non-collinear wave mixing at a contact interface," Journal of Sound and Vibration, vol. 468, no. 3, Article ID 115078, 22 pages, 2020.

[28] K. Manktelow, R. K. Narisetti, M. J. Leamy, and M. Ruzzene, "Finite-element based perturbation analysis of wave propagation in nonlinear periodic structures," Mechanical System and Signal Processing, vol. 39, no. 1-2, pp. 32-46, 2013.

[29] K. Manktelow, M. J. Leamy, and M. Ruzzene, "Comparison of asymptotic and transfer matrix approaches for evaluating intensity-dependent dispersion in nonlinear photonic and phononic crystals," Wave Motion, vol. 50, no. 3, pp. 494-508, 2013.

[30] P. Blanloeuil, L. R. F. Rose, M. Veidt, and C. H. Wang, "Analytical and numerical modelling of wave scattering by a 
linear and nonlinear contact interface," Journal of Sound and Vibration, vol. 456, pp. 431-453, 2019.

[31] T. Nam, T. Lee, C. Kim, K.-Y. Jhang, and N. Kim, "Harmonic generation of an obliquely incident ultrasonic wave in solidsolid contact interfaces," Ultrasonics, vol. 52, no. 6, pp. 778-783, 2012.

[32] C. Pecorari, "Nonlinear interaction of plane ultrasonic waves with an interface between rough surfaces in contact," The Journal of the Acoustical Society of America, vol. 113, no. 6, pp. 3065-3072, 2003.

[33] Y. Zhao, Y. Qiu, L. J. Jacobs, and J. Qu, "A micromechanics model for the acoustic nonlinearity parameter in solids with distributed micro cracks," AIP Conference Processing, vol. 1706, no. 1, 9 pages, Article ID 060001, 2016.

[34] Y. Zhao, F. Li, P. Cao et al., "Generation mechanism of nonlinear ultrasonic Lamb waves in thin plates with randomly distributed micro-cracks," Ultrasonics, vol. 79, pp. 60-67, 2017.

[35] P. Blanloeuil, A. Meziane, and C. Bacon, "Numerical study of nonlinear interaction between a crack and elastic waves under an oblique incidence," Wave Motion, vol. 51, no. 3, pp. 425-437, 2014.

[36] P. Blanloeuil, A. J. Croxford, and A. Meziane, "Numerical and experimental study of the nonlinear interaction between a shear wave and a frictional interface," The Journal of the Acoustical Society of America, vol. 135, no. 4, pp. 1709-1716, 2014.

[37] P. Blanloeuil, L. R. F. Rose, M. Veidt, and C. H. Wang, "Time reversal invariance for a nonlinear scatterer exhibiting contact acoustic nonlinearity," Journal of Sound and Vibration, vol. 417, no. 17, pp. 413-431, 2018.

[38] X. Wan, P. W. Tse, G. H. Xu, T. F. Tao, and Q. Zhang, "Analytical and numerical studies of approximate phase velocity matching based nonlinear S0 mode Lamb waves for the detection of evenly distributed microstructural changes," Smart Materials and Structures, vol. 25, Article ID 045023, 20 pages, 2016.

[39] Z. Nie, K. Wang, and M. Zhao, "Application of wavelet and EEMD joint denoising in nonlinear ultrasonic testing of concrete," vol. 2018, Article ID 7872036, 11 pages, 2018.

[40] P. Blanloeuil, A. Meziane, A. N. Norris, and C. Bacon, "Analytical extension of finite element solution for computing the nonlinear far field of ultrasonic waves scattered by a closed crack," Wave Motion, vol. 66, pp. 132-146, 2016.

[41] K. Kazushi and I. Yasuaki, "A finite difference method for elastic wave scattering by a planar crack with contacting faces," Wave Motion, vol. 52, pp. 120-137, 2015.

[42] X. Sun, H. Liu, Y. Zhao, J. Qu, M. Deng, and N. Hu, "The zerofrequency component of bulk waves in solids with randomly distributed micro-cracks," Ultrasonics, vol. 107, Article ID 106172, 8 pages, 2020.

[43] K. Kawashima, R. Omote, T. Ito, H. Fujita, and T. Shima, "Nonlinear acoustic response through minute surface cracks: FEM simulation and experimentation," Ultrasonics, vol. 40, no. 1-8, pp. 611-615, 2002.

[44] L. Vergara, R. Miralles, J. Gosálbez et al., "NDE ultrasonic methods to characterise the porosity of mortar," NDT \& $E$ International, vol. 34, no. 8, pp. 557-562, 2001. 